\title{
Diversity of Adsorbed Hydrogen on the TiC (001) Surface at High Coverages
}

\author{
Juan José Piñero, ${ }^{\dagger}$ Pedro J. Ramírez, ${ }^{\star}$ Stefan T. Bromley, ${ }^{\dagger,}$ Francesc Illas, ${ }^{\dagger}$ Francesc Viñes, ${ }^{* \dagger}$ \\ and José A. Rodriguez ${ }^{*}$, \\ † Departament de Ciència dels Materials i Química Física \& Institut de Química Teòrica i \\ Computacional (IQTCUB), Universitat de Barcelona, c/ Martí i Franquès 1-11, 08028 Barcelona, \\ Spain \\ $\$$ Facultad de Ciencias, Universidad Central de Venezuela, Apartado 20513, Caracas 1020-A, \\ Venezuela \\ $\S$ Institució Catalana de Recerca i Estudis Avançats (ICREA), Passeig de Lluís Companys, 23, \\ 08010 Barcelona (Spain) \\ "Chemistry Department, Brookhaven National Laboratory, Upton, NY 11973, United States \\ * Corresponding authors: francesc.vines@ub.edu, rodrigez@,bnl.gov
}

\begin{abstract}
The catalyzed dissociation of molecular hydrogen on the surfaces of diverse materials is currently widely studied due to its importance in a broad range of hydrogenation reactions that convert noxious exhaust products and/or greenhouse gases into added-value greener products such as methanol. In the search for viable replacements for expensive late transition metal catalysts $\mathrm{TiC}$ has been increasingly investigated as a potential catalyst for $\mathrm{H}_{2}$ dissociation. Here, we report on a combination of experiments and density functional theory calculations on the well-defined TiC(001) surface revealing that multiple $\mathrm{H}$ and $\mathrm{H}_{2}$ species are available on this substrate, with different binding configurations and adsorption energies. Our calculations predict an initial occupancy of $\mathrm{H}$ atoms on surface $\mathrm{C}$ atom sites, which then enables the subsequent stabilization of $\mathrm{H}$ atoms on top of surface Ti atoms. Further $\mathrm{H}_{2}$ can be also molecularly adsorbed over Ti sites. These theoretical predictions are in full accordance with information extracted from X-ray photoemission spectroscopy and temperature-programmed desorption experiments. The experimental results show that at high coverages of hydrogen there is a reconstruction of the $\mathrm{TiC}(001)$ surface which facilitates the binding of the adsorbate.
\end{abstract}




\section{Introduction}

The interaction of molecular hydrogen with titanium carbide (TiC) is an important topic in catalysis, solid-state chemistry, electrochemistry, and materials science. ${ }^{1-9} \mathrm{TiC}$ displays high activity for the hydrogenations of ethylene and $\mathrm{CO}_{2} .13 \%$ In principle, ethylene and $\mathrm{CO}_{2}$ molecules prefer to adsorb on the carbon sites of $\mathrm{TiC},{ }^{3,10}$ leaving the $\mathrm{Ti}$ sites free for the dissociation of $\mathrm{H}_{2}$ and subsequent hydrogenation reactions. In the case of $\mathrm{CO}_{2}$, an attack of $\mathrm{H}$ to form a $\mathrm{HOCO}$ intermediate is a key step that precedes the formation of $\mathrm{CO}$ and its subsequent hydrogenation to methanol. ${ }^{3,4}$ To carry out these reactions in an efficient way, hydrogen atoms must be present on the TiC surface while simultaneously displaying a high activity and mobility. Therefore, it is important to study the interaction of $\mathrm{H}$ with the carbide surface as a function of coverage and establish how much hydrogen a nearly stoichiometric TiC system can adsorb and/or absorb In principle, hydrogen can be present at different TiC sites: On the surface, ${ }^{3,4}$ in the bulk, ${ }^{5-9}$ in interstitial positions, ${ }^{11}$ physisorbed ${ }^{12}$ and forming a Kubas structure. ${ }^{13,14}$ In this aspect, TiC can be regarded as a textbook example within the family of transition metal carbide (TMC) materials. ${ }^{14,15}$

It has also been shown that $\mathrm{TiC}$ can be useful as a material for hydrogen storage, although the low gravimetric content prevents its technological use. ${ }^{6-9}$ Under electrochemical conditions $\mathrm{H}$ is found to be absorbed on defective $\mathrm{TiC}_{\mathrm{x}}$ compounds. ${ }^{6,7}$ As the $\mathrm{TiC}_{\mathrm{x}}$ composition progressively deviates from a 1:1 stoichiometry, the insertion of $\mathrm{H}$ and/or $\mathrm{H}_{2}$ into the substrate lattice increases linearly, where $\mathrm{H} / \mathrm{H}_{2}$ species occupy carbon vacancies, ${ }^{7,} 8$ a point that also occurs in heterogeneous catalysis working conditions. ${ }^{16,17}$ Studies using X-ray diffraction (XRD)6?7 have shown that $\mathrm{TiC}$ allows the insertion of hydrogen within its structure, but only under two conditions. First, the carbide should have a high concentration of $\mathrm{C}$ vacancies with a composition 
in the range of $\mathrm{TiC}_{0.6-0.7}$, thus likely containing $\mathrm{C}$ vacancies in the bulk matrix. Second, carbon vacancies must be ordered, leaving the (111) planes as empty as possible, as disordered carbide structures do not allow significant hydrogen insertion. Results of density functional theory (DFT) based calculations suggest that nanoparticles of titanium carbide, such as $\operatorname{Ti}_{8} \mathrm{C}_{12}$ and $\mathrm{Ti}_{14} \mathrm{C}_{13}$, have potential for hydrogen storage at near ambient conditions. 5 The titanium atoms in these systems catalyze the dissociation of $\mathrm{H}_{2}$ and are capable of coordinating with multiple hydrogen ligands. ${ }^{5}$ Inelastic and elastic neutron scatterings have shown the presence of molecular hydrogen trapped in two-dimensional layered titanium carbide-based MXenes $\left(\mathrm{Ti}_{3} \mathrm{C}_{2} \mathrm{~T}_{\mathrm{x}}\right.$, where $\mathrm{T}_{\mathrm{x}}$ represents a surface terminating species, including $\mathrm{H}, \mathrm{O}, \mathrm{OH}$, and F). ${ }^{9}$ The trapped $\mathrm{H}_{2}$ occupies small cavities in the structure of the MXenes and exhibits a high mobility. ${ }^{9}$ Previous studies have shown that $\mathrm{H}_{2}$ adsorption in TiC occurs, but the $\mathrm{H}_{\mathrm{x}}$ species present on this carbide or how the surface becomes covered as the $\mathrm{H}_{2}$ pressure increases were hitherto not reported. In general, $\mathrm{H}_{2}$ and/or $\mathrm{H}$ species are particularly important species in heterogeneously catalyzed processes, ${ }^{18}$ such as fossil fuel refinement, hydrogenation of alkenes, ${ }^{19}$ and the production of other diverse chemicals compounds.

Herein, surface and subsurface $\mathrm{H}_{\mathrm{x}}$ species are systematically studied on TiC by both theory and experiments. Note that TiC is a system that is representative of TMCs with 1:1 stoichiometry. Specifically, we sample $\mathrm{H}_{2}$ on $\mathrm{TiC}(001)$ surfaces with increasing hydrogen doses and pressures by combining both X-ray photoelectron spectroscopy (XPS) and temperature programmed desorption (TPD) with DFT based calculations. Hereby, the interaction of $\mathrm{H}_{2}$ with the well-defined $\mathrm{TiC}(001)$ surface is determined considering a wide range of molecular and atomic adsorption scenarios. The desorption of the adsorbed $\mathrm{H}_{2}$ molecule is observed over a broad range of temperatures $(\sim 150 \mathrm{~K})$, consistent with a large variation in the $\mathrm{H}$ adsorption 
energy with respect to coverage, as predicted by the DFT based simulations. At room temperature, more than a monolayer of hydrogen can adsorb on the stoichiometric TiC(001) surface. Moreover, our experimental and theoretical studies point to a $\mathrm{H}$-induced reversible reconstruction of the carbide surface that facilitates the dissociation of $\mathrm{H}_{2}$ and the binding of hydrogen in a diversity of ways.

\section{Experimental and theoretical methods}

TPD and XPS studies. The $\mathrm{TiC}(001)$ single crystal was cleaned following procedures reported in the literature. ${ }^{20-22}$ The cleaning methodology led to a clear $(1 \times 1)$ diffraction pattern by lowenergy electron diffraction (LEED) and no surface impurities detected by photoemission or XPS. The crystal growers estimated a $\mathrm{TiC}_{0.95-0.98}$ stoichiometry for the bulk of the sample, and, after cleaning, our quantitative XPS results showed surfaces with essentially a Ti/C ratio of one. ${ }^{22}$ For surfaces prepared in this way, images of scanning tunneling microscopy (STM) gave a square crystal lattice with terraces that were $400-650 \AA$ wide, separated by single and double step heights. For a sample with these characteristics, no penetration of $\mathrm{H}_{2}$ or $\mathrm{H}$ into the bulk is to be expected. $^{8}$

The experiments were carried out in an ultrahigh-vacuum (UHV) chamber that has attached a high-pressure cell or batch reactor. ${ }^{3,4}$ The sample could be transferred between the reactor and UHV chamber without exposure to air. The UHV chamber (base pressure $1 \times 10^{-10}$ Torr) was equipped with instrumentation for XPS, LEED, ion-scattering spectroscopy (ISS), and TPD. ${ }^{3,4}$ The $\mathrm{TiC}(001)$ was exposed to $\mathrm{H}_{2}(>99.999 \%$ purity) at $300 \mathrm{~K}$ in the UHV chamber or in the high pressure reactor. After exposure to hydrogen, the TPD spectra were acquired with a heating rate of $2 \mathrm{~K} / \mathrm{s}$. The amount of hydrogen under each TPD peak was calibrated using TPD 
data for a saturation coverage of hydrogen on $\mathrm{Pt}(111) .{ }^{23}$ Coverages of hydrogen are reported with respect to the total number of $\mathrm{Ti}$ and $\mathrm{C}$ atoms in a $\mathrm{TiC}(001)$ surface. In the TPD experiments, at a nominal coverage of one monolayer (ML), all the atoms of the carbide surface are bound to one $\mathrm{H}$ atom.

DFT based calculations. Periodic DFT based calculations using suitable supercell models representing the systems of interest were carried out to investigate the role of the TiC surface on atomic $(\mathrm{H})$ and molecular hydrogen $\left(\mathrm{H}_{2}\right)$ adsorption. Only the TiC $(001)$ surface was considered as previous studies already demonstrated that this surface is the most stable one for $\mathrm{TiC}$ and other TMC compounds. ${ }^{24}$ This surface was modeled by periodically repeated two-dimensional slabs with a separating vacuum region of $10 \AA$, which was large enough to avoid spurious interactions between slabs periodically repeated along the surface direction. ${ }^{13,15}$ The slab was constructed using the lattice parameter taken from the optimized bulk TiC crystal. ${ }^{25}$ The slab contains four atomic layers; the two outermost ones were allowed to fully relax, whereas the two bottom layers were fixed at bulk positions to simulate the bulk. To represent the (001) surface a $(2 \sqrt{2} \times 2 \sqrt{2}) \mathrm{R} 45^{\circ}$ supercell was employed. The supercell contains a total of $64(32 \mathrm{C}$ and $32 \mathrm{Ti})$ atoms.

All calculations were carried out using the Vienna Ab Initio simulation package VASP code. $^{26}$ The Perdew-Burke-Ernzerhof $(\mathrm{PBE})^{27}$ exchange-correlation functional was chosen and the effect of dispersive forces was described by the D3 dispersion correction developed by Grimme (PBE-D3) ${ }^{28}$ The effect of core electrons on the valence electron density was taken into account through the projected augmented wave method of Blöchl, ${ }^{29}$ as implemented by Kresse and Joubert, with $2 s^{2} 2 p^{2}$ and $2 p^{6} 3 d^{3} 3 s^{1}$ for $\mathrm{C}$ and $\mathrm{Ti}$ atoms, respectively. ${ }^{30}$ The valence electron 
density was expanded in a plane wave basis set with a kinetic energy cut-off of $415 \mathrm{eV}$. Monkhorst-Pack k-point schemes ${ }^{31}$ of $9 \times 9 \times 1$ dimensions were used for bulk and surface slab calculations. A conjugated-gradient algorithm was employed for the atomic structure optimization, together with a force convergence criterion of $0.01 \mathrm{eV} \AA^{-1}$. The tetrahedron smearing method with Blöchl corrections with a smearing width of $0.2 \mathrm{eV}$ was used to determine partial occupancies for each orbital and total energies were then extrapolated to $0 \mathrm{~K}$ (no smearing). The electronic relaxation convergence criterion was set to $10^{-5} \mathrm{eV}$.

The sequential adsorption energy $\left(E_{a d s}\right)$ of a $n^{\text {th }}$ adsorbed $\mathrm{H}$ atom was calculated as:

$$
E_{a d s}=\left(E_{n \cdot H / T i C}-\left(E_{H}-E_{(n-1) \cdot H / T i C}\right)\right)
$$

where $E_{n \cdot H / T i C}$ is the energy of the $n \cdot \mathrm{H}$ atoms adsorbed on the surface, $E_{H}$ is the energy of an isolated $\mathrm{H}$ atom, $n$ is the number of $\mathrm{H}$ atoms in the system, and $E_{(n-1) \cdot H / T i C}$ is the energy of the relaxed TiC surface containing $n-1 \mathrm{H}$ atoms, each in its optimal position. With this definition, a more negative the $E_{a d s}$ value indicates a stronger adsorption. $E_{H}$ is obtained by placing the atom in a broken symmetry unit cell of $9 \times 10 \times 11 \AA$ dimensions and carrying out a $\Gamma$-point optimization. Notice that when ${ }^{1} / 2 \cdot \mathrm{H}_{2}$ is used as an energy reference, i.e., using $E_{H_{2}}$ instead of $E_{H}$ and calculated likewise, the obtained $E_{a d s}$ get systematically reduced by $2.26 \mathrm{eV}$ either at PBE or PBE-D3 levels.

In order to ascertain whether the $\operatorname{TiC}(001)$ surface is suitable to dissociate $\mathrm{H}_{2}$, the transitions state for the $\mathrm{H}_{2}$ splitting reaction step was obtained by using the climbing image nudged elastic band (Cl-NEB) $)^{32}$ procedure. To characterize the thus obtained candidate transition state (TS), an analysis of vibrational frequencies was performed. Here, in the construction and diagonalization of the Hessian matrix built from finite difference displacements of $0.03 \AA$, only the degrees of freedom of the adsorbed $\mathrm{H}_{\mathrm{x}}$ were considered. A well-defined TS is confirmed 
when only a single imaginary frequency is obtained.

To study the desorption process, one could use statistical thermodynamics coupled to transition state theory (TST) to obtain adsorption and desorption rate estimates. ${ }^{33,34}$ However, the prediction of the TPD peak is more involved because it corresponds to the temperature at which the desorption rate is maximum. Therefore, temperature of maximum desorption rate, $T_{d}$, of the adsorbed $\mathrm{H}$ atoms has been estimated by using the broadly employed Redhead equation. ${ }^{35}$ The desorption is assumed to be a second-order reaction, given that $\mathrm{H}$ adatoms have to diffuse in order to recombine into a $\mathrm{H}_{2}$ molecule prior to desorption. Thus, the employed Redhead equation is

$$
E_{\text {des }}=R T_{d}^{2} \cdot\left(\frac{\theta A}{\beta} \exp ^{\frac{-E_{d e s}}{R T_{d}}}\right)
$$

where $R$ is the ideal gases constant, $\theta$ is the surface coverage, $A$ is a rate pre-exponential factor, which can be estimated from TST and has values in a range between $10^{8}$ and $10^{13}$ under the general validity of Redhead equation, $\beta$ is the heating rate of $2 \mathrm{~K} / \mathrm{s}$ in accordance to TPD spectra, and $E_{d e s}$ is the desorption activation energy, here quantified as minus the $E_{a d s}$. In the present work the pre-exponential factor is chosen to cover a broad range and thus minimize possible effects coming from the approximations involved in TST.

\section{Results and Discussion}

Binding and desorption of hydrogen: TPD and XPS spectra. Figure 1 shows a set of TPD spectra recorded after different exposures of molecular hydrogen to a clean $\operatorname{TiC}(001)$ surface at $300 \mathrm{~K}$. The uppermost spectrum in Figure 1 was recorded after exposing the sample at $300 \mathrm{~K}$ to 1 Torr of $\mathrm{H}_{2}$ for 5 minutes in a micro-reactor. The three lower spectra of the figure correspond to UHV exposures of 5, 50, and 150 Langmuir (L). Desorption of hydrogen is observed at 
temperatures between 320 and $430 \mathrm{~K}$. A weak peak is observed around $410 \mathrm{~K}$ and could come from adsorption and dissociation of $\mathrm{H}_{2}$ on defect sites of the $\mathrm{TiC}(001)$ surface. The peaks at 370 and $340 \mathrm{~K}$ are probably associated with hydrogen bound to ideal terrace sites of TiC(001). For doses under UHV conditions, saturation in the $\mathrm{H}$ coverage of 0.6-0.7 ML was seen upon $\mathrm{H}_{2}$ exposures above $100 \mathrm{~L}$. As we will see below, this saturation coverage for UHV dosing is in good agreement with the corresponding coverage calculated using DFT for $\mathrm{H}$ on a perfect $\mathrm{TiC}(001)$ surface at $300 \mathrm{~K}$. A higher $\mathrm{H}$ coverage of 1.4-1.6 ML was seen upon exposure of the sample to 1 Torr of $\mathrm{H}_{2}$ and led to a hydrogen desorption peak at 320-330 K. This much higher coverage of hydrogen is probably associated with a reconstruction of the carbide surface. ${ }^{34,35}$ 


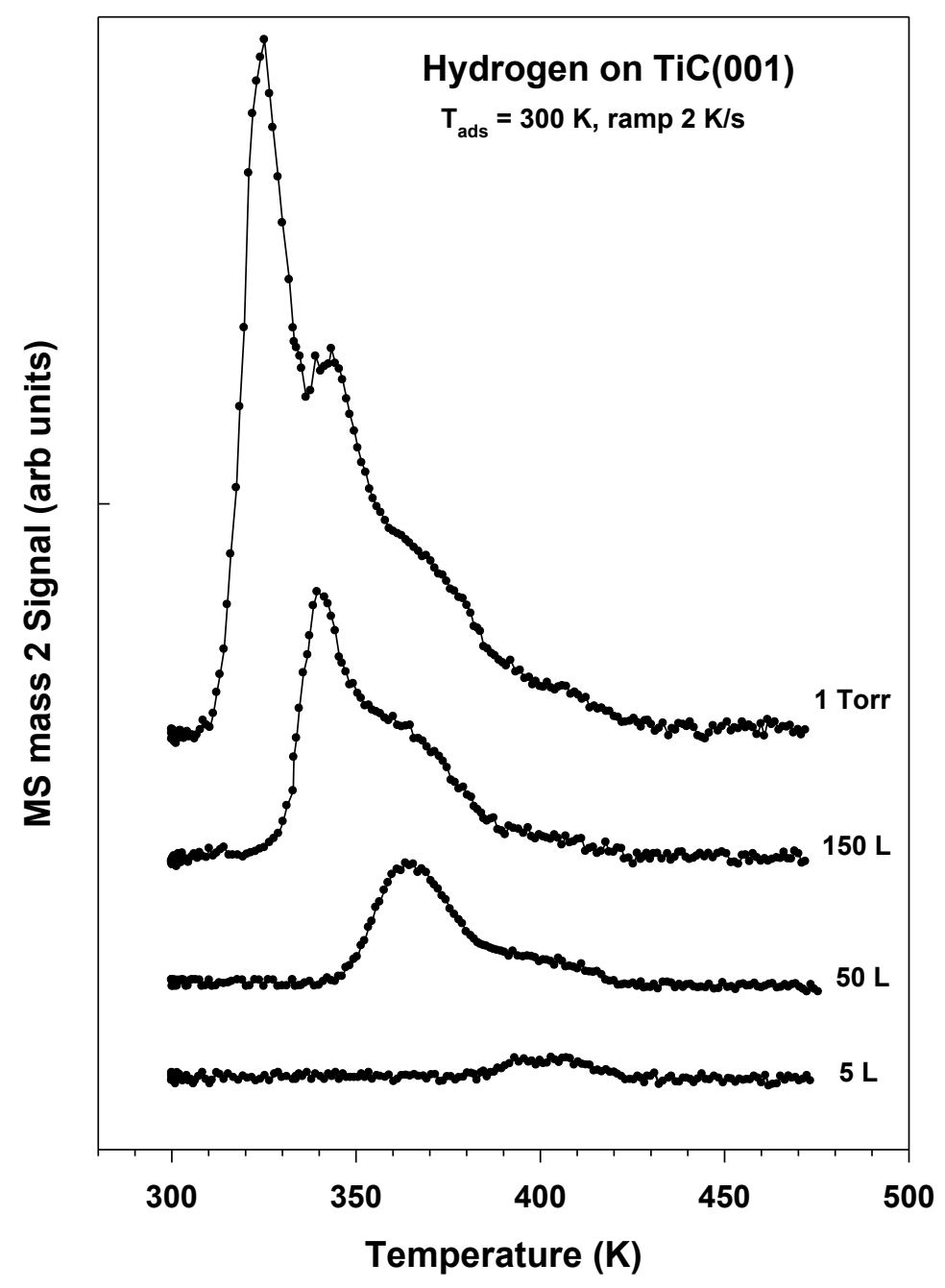

Figure 1. $\mathrm{H}_{2}$-Thermal desorption spectra collected after dosing molecular hydrogen with 5,50 , and 150 Langmuir $(\mathrm{L})$ to a $\mathrm{TiC}(001)$ surface at $300 \mathrm{~K}$. The top spectrum corresponds to a surface exposed to 1 Torr of $\mathrm{H}_{2}$ for 5 minutes.

Desorption spectra collected after trapping $\mathrm{H}_{2}$ in rough powders of TiC show evolution of $\mathrm{H}_{2}$ in a medium-temperature $(330-573 \mathrm{~K})$ and a high-temperature range $(723-1000 \mathrm{~K}){ }^{36,37}$ The high-temperature desorption features are usually assigned to hydrogen trapped inside the titanium carbide and/or bound to highly defective sites of the substrate. We did not observe these high temperature features in our TPD studies. On a TiC(001) substrate with a 1:1 stoichiometric 
ratio, no significant penetration of hydrogen into the bulk of the sample is expected. ${ }^{8}$ Furthermore, the theoretical calculations to be discussed in the next section show that hydrogen atoms or molecules are not stable in the interstitial sites of a perfect $\mathrm{TiC}(001)$ lattice. However, a reconstruction in the lattice of the carbide could produce sites where hydrogen binds well..$^{34,35}$ The large adsorbate coverages ( $>1 \mathrm{ML}$ ) seen in experiments for Torr doses of $\mathrm{H}_{2}$ are probably associated with the formation of compounds on the surface of the sample. Figure 2 shows C $1 s$ XPS spectra collected before and after exposing a $\mathrm{TiC}(001)$ surface to $1 \mathrm{Torr}$ of $\mathrm{H}_{2}$ at $300 \mathrm{~K}$. In the clean sample one sees the $\mathrm{C} 1 s$ peak expected for titanium carbide. ${ }^{22}$ Upon contact with 1 Torr of $\mathrm{H}_{2}$, a new peak appears at near $283.5 \mathrm{eV}$ that indicates the formation of $\mathrm{CH}_{\mathrm{x}}$ groups on the surface of the carbide. ${ }^{38}$ This peak was not seen for the UHV doses of $\mathrm{H}_{2}$. Under a high pressure of $\mathrm{H}_{2}$, the surface of the carbide appears to generate the $\mathrm{CH}_{\mathrm{x}}$ groups and accommodate an extra amount of hydrogen not bound to $\mathrm{C}$. The $\mathrm{CH}_{\mathrm{x}}$ decomposed when the surface was heated to $500 \mathrm{~K}$. Thus, the data point to a reversible mechanism for the storage of hydrogen on the surface of the carbide. 


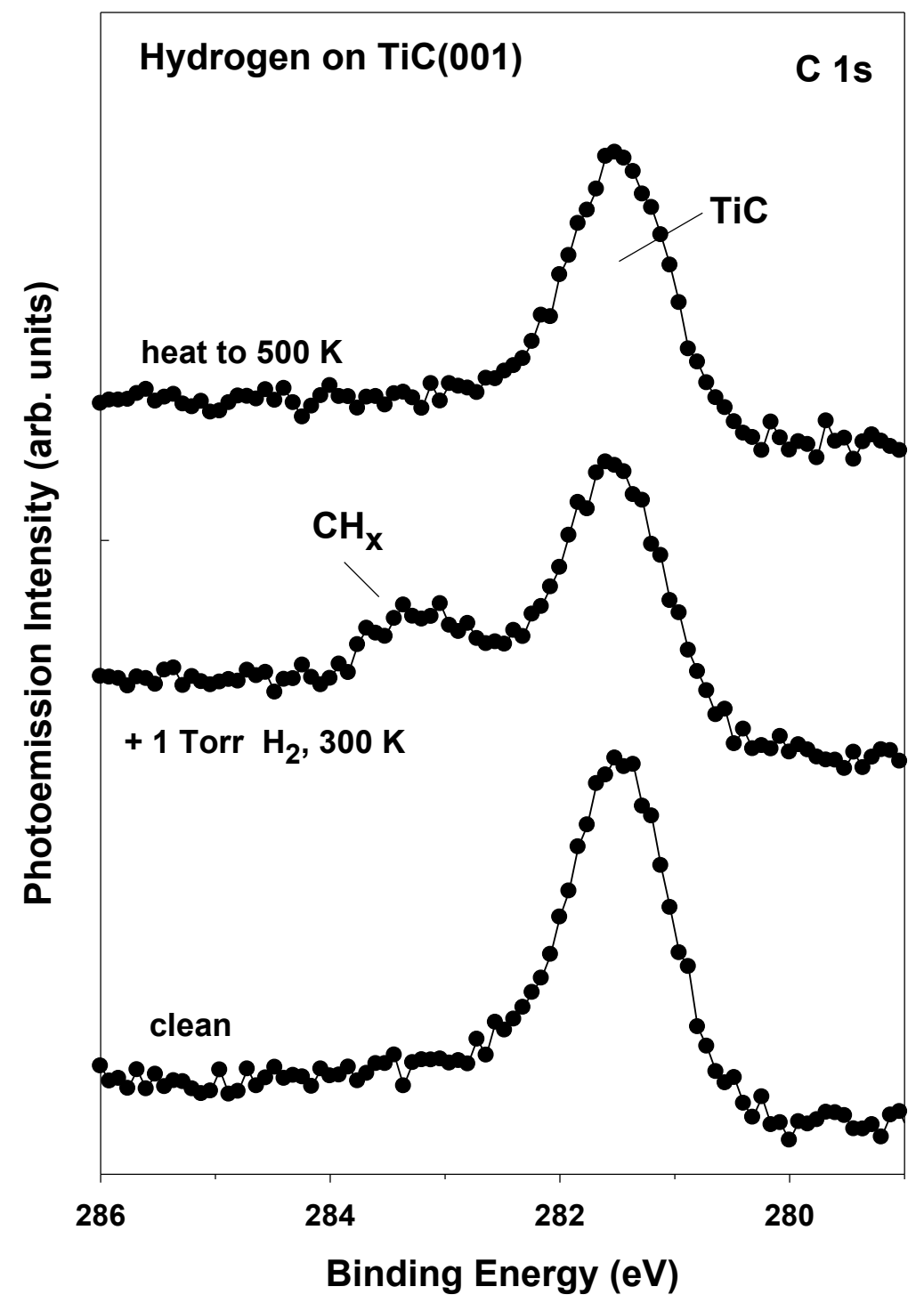

Figure 2. C $1 s$ XPS spectra for clean $\mathrm{TiC}(001)$, bottom, a surface exposed at $300 \mathrm{~K}$ to 1 Torr of $\mathrm{H}_{2}$ for 5 minutes, middle, and the $\mathrm{H}_{2} / \mathrm{TiC}(001)$ system heated to $500 \mathrm{~K}$, top.

Hydrogen adsorption: DFT based calculations. First, atomic $H$ adsorption on $\operatorname{TiC}(001)$ surface was studied by considering the adsorption of a single $\mathrm{H}$ atom on the above-described slab model. This model system implies a small $\mathrm{H}$ coverage of 0.125 monolayers (ML). Notice that, at variance with the $\mathrm{Pt}(111)$ reference for $\mathrm{H}$ coverage, here a monolayer is defined according to the 
number of surface titanium atoms (or equally well to the same number of surface carbon atoms). $\mathrm{H}$ adsorption was explored on six different sites of the $\mathrm{TiC}(001)$ surface, see Figure $\mathrm{S} 1$ of the Supplementary Information.

The results from the DFT-based calculations show that the most stable adsorption configuration is the C-top site with adsorption energy of $-2.73 \mathrm{eV}$ predicted by the PBE functional, and $-2.83 \mathrm{eV}$ for calculations using PBE-D3, see Table 1 in the Supplementary Information. In a second step, $\mathrm{H}_{2}$ adsorption was explored considering two different orientations with respect to the surface, with the molecular axis either vertical or horizontal with respect to the surface. Thus, $\mathrm{H}_{2}$ adsorption was considered by exploring 19 configurations where six positions were vertical and 13 were horizontal, see Figure S2, in the Supplementary Information. In accordance with previous reports in the literature, ${ }^{13}$ the most energetically favorable position for $\mathrm{H}_{2}$ adsorption is the $\mathrm{C}$-top position, adopting a so-called Kubas mode of adsorption. ${ }^{13,14}$ This situation corresponds to a noticeable elongation of the $\mathrm{H}-\mathrm{H}$ bond length, although with no bond breaking, and with a PBE adsorption energy value of $-0.46 \mathrm{eV}(-0.66 \mathrm{eV}$ at PBE-D3). The adsorption energy values thus obtained are similar to those reported in a previous study on $\mathrm{TiC}(001)$ using the PW91 functional ${ }^{39}$ with a $\mathrm{H}$ adsorption energy of $-2.60 \mathrm{eV}$, and $\mathrm{a}_{2}$ adsorption energy of $-0.48 \mathrm{eV} .{ }^{13}$ The other less energetically favorable minima are shown in Tables S1 and S2 of the Supplementary Information.

The $\mathrm{H}_{2}$ dissociation kinetics for a $\mathrm{H}_{2} \mathrm{C}$-top Kubas bonding on $\mathrm{TiC}(001)$ has been examined by searching for the reaction step transition state, and the associated activation energy barrier. To this end, the reaction path, shown in Figure 3, is sketched with $\mathrm{H}_{2}$ being initially molecularly adsorbed on a C-top, and then breaking into two $\mathrm{H}$ atoms, which both adsorb on neighboring C-top sites. The reaction profile reveals an energy barrier of $0.49 \mathrm{eV}$ only, while the 
effective energy barrier with respect to the energy reference of non-interacting $\mathrm{TiC}(001)$ and $\mathrm{H}_{2}$ is negligible $(0.03 \mathrm{eV})$.

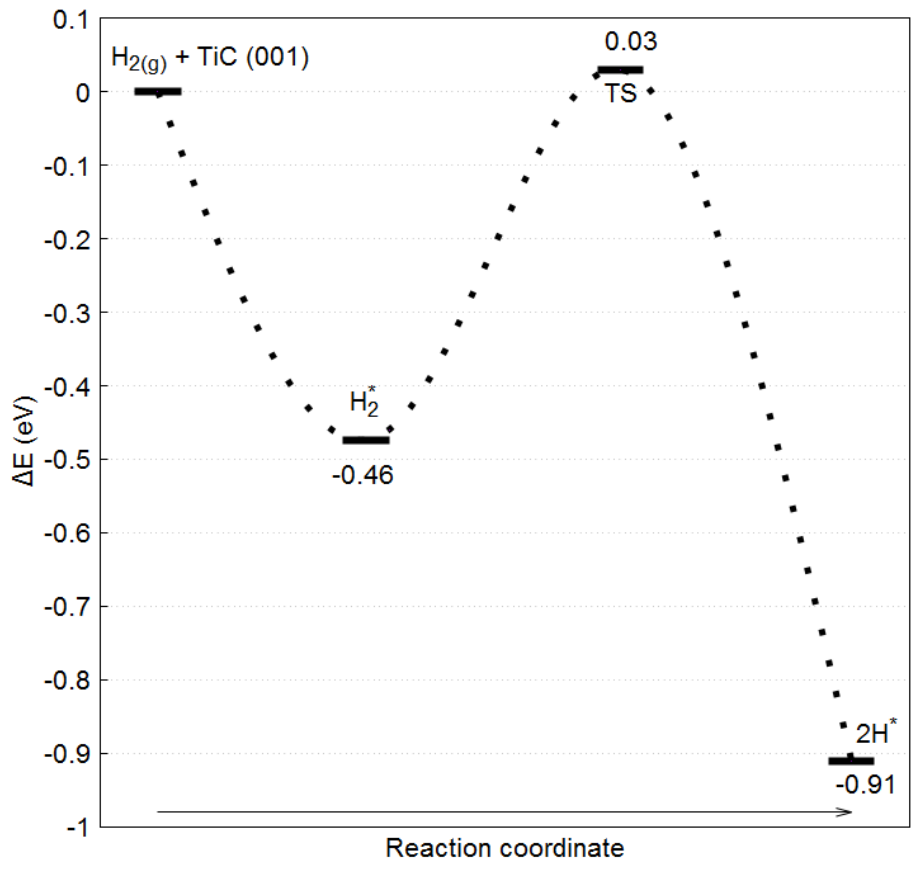

Figure 3. Reaction profile of $\mathrm{H}_{2}$ adsorption and scission on the $\mathrm{TiC}(001)$ surface.

The TS has only one imaginary frequency of $1377 \mathrm{~cm}^{-1}$, corresponding to a $\mathrm{H}_{2}$ separation mode, thus confirming its true saddle point nature. These results confirm that $\mathrm{H}_{2}$ dissociation is possible and likely under catalytic working conditions given the small energy barrier of only $0.49 \mathrm{eV}$, thus no rate limiting step of $\mathrm{H}$ adsorption on $\mathrm{TiC}(001)$ surface is expected.

The $\mathrm{H}$ diffusion was also studied with respect to two different situations: When $\mathrm{H}$ diffuses to a vicinal $\mathrm{C}$ atom, or when $\mathrm{H}$ diffuses to more distant $\mathrm{C}$ atoms, see Figure 4. 


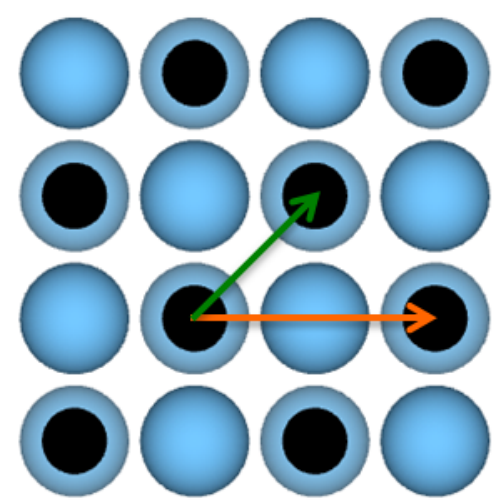

Figure 4. $\mathrm{H}$ diffusion studies. Blue balls represent Ti atoms, black balls are $\mathrm{C}$ atoms. The green arrow indicates the diffusion path to vicinal $\mathrm{C}$ atom while orange arrow shows the path to a more distant $\mathrm{C}$ atom.

The results obtained show that the energy barrier for $\mathrm{H}$ atoms to diffuse towards a vicinal $\mathrm{C}$ atom $(0.86 \mathrm{eV})$ is much lower than diffusion to more distant $\mathrm{C}$ atoms $(2.11 \mathrm{eV})$. Considering these values, when $\mathrm{H}_{2}$ dissociation occurs, the $\mathrm{H}$ atoms are predicted to be adsorbed on two vicinal $\mathrm{C}$ atoms.

Next, the adsorption energy at different $\mathrm{H}$ coverages, from 0.125 to $1 \mathrm{ML}$ was studied. For each $\mathrm{H}$ coverage considered many $\mathrm{H}$ atoms placements were examined, including vicinal and remote placements. In all cases, the $\mathrm{H}$ adsorption is energetically favorable, as can also be seen in Figure 5. Figure $\mathrm{S} 3$ of the Supplementary Information shows the sequence of $\mathrm{H}$ atom filling on the $\mathrm{TiC}(001)$ surface. 


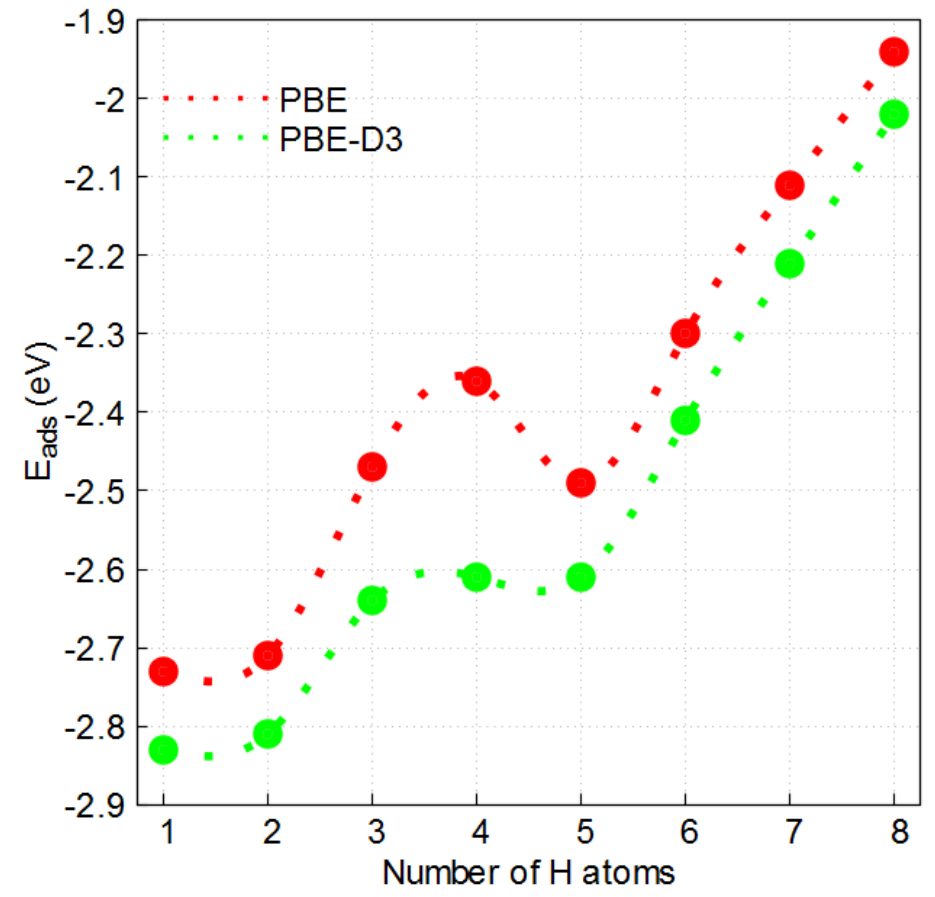

Figure 5. Adsorption energies $\left(\mathrm{E}_{\mathrm{ads}}\right.$, in $\left.\mathrm{eV}\right)$ for different $\mathrm{H}$ atom coverages on $\mathrm{TiC}(001)$ surface.

The results show that the sequential $E_{a d s}$ per $\mathrm{H}$ atom decreases as more $\mathrm{H}$ atoms are added to the surface. These values reduce by $2.26 \mathrm{eV}$ when using half the energy of a $\mathrm{H}_{2}$ molecule as a reference, see Table S4. Taking this into consideration, most of the $\mathrm{H}$ adsorptions are thermodynamically favorable, but a full occupancy of all surface $\mathrm{C}$ positions would be only achievable under a certain $\mathrm{H}_{2}$ partial pressure.

The observed decrease of the $E_{a d s}$ with coverage is as expected, since at this higher coverage situation the electronic repulsion between the adsorbed atoms increases thus causing the adsorption energy to decrease. An interesting feature of Figure 5 is that the adsorption energy is not monotonically decreased along the explored $\mathrm{H}$ coverage range. Attention is drawn in Figure 5 as when adding the fifth $\mathrm{H}$, the system gets extra-stabilized. This phenomenon may be related to the key role of electrostatic forces of the system and, hence, it has been further studied. To further evaluate this situation four $\mathrm{H}$ atoms were adsorbed on vicinal on $\mathrm{C}$-top sites, and a 
fifth $\mathrm{H}$ atom was added over diverse positions, either on Ti-top in three different places, or on a nearby $\mathrm{C}$ atom, see sites in Figure 6.

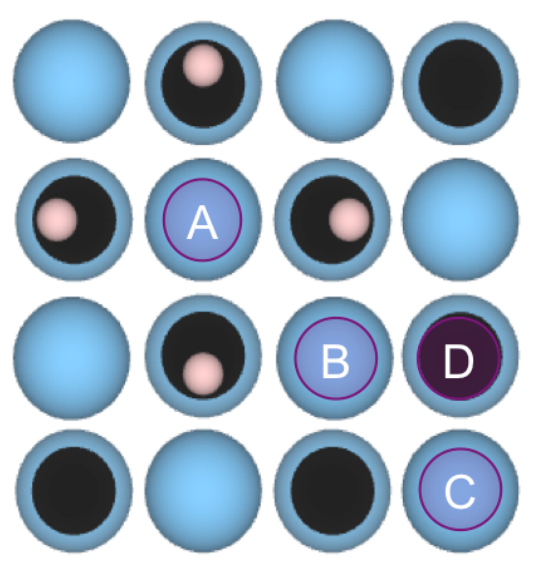

Figure 6. Top view of the tested adsorption sites (A-D) of a fifth $\mathrm{H}$ atom when four $\mathrm{H}$ are simultaneously adsorbed vicinal on C-top sites. Blue balls represent Ti atoms, black balls are $\mathrm{C}$ atoms

Here incremental adsorption energies are obtained as:

$$
E_{\text {ads }}=\left(E_{H / T i C}-\left(E_{H}-E_{T i C+4 H}\right)\right)
$$

where $E_{H / T i C}$ is the energy of the surface with five $\mathrm{H}$ atoms, and $E_{T i C+4 H}$ is the energy of the surface with $4 \mathrm{H}$ atoms adsorbed on vicinal $\mathrm{C}$ atoms. The results for the adsorption energy values for the fifth $\mathrm{H}$ atom are reported in Table 1.

Results show that the fifth $\mathrm{H}$ prefers to adsorb on C-top than on Ti-top, with the adsorption energies being -2.49 and $-1.95 \mathrm{eV}$, respectively. Not all Ti-top positions are equivalent, and the adsorption energy may vary by up to $\sim 0.9 \mathrm{eV}$ depending.. The central Ti-top position (A), for example, has a much larger adsorption energy than other two Ti-top positions (B and C), see Figure 6. 
Table 1. Adsorption energies for the fifth $\mathrm{H}$ atom on $\mathrm{TiC}(001)$ on different sites as shown in Figure 6. All values are given in $\mathrm{eV}$.

\begin{tabular}{|c|c|c|c|c|}
\hline \multirow{2}{*}{$E_{\text {ads }}^{H}$} & \multicolumn{3}{|c|}{ Ti-top } & C-top \\
\cline { 2 - 5 } & Situation A & Situation B & Situation C & Situation D \\
\hline PBE & -1.95 & -1.62 & -1.06 & -2.49 \\
\hline PBE-D3 & -2.03 & -1.69 & -1.12 & -2.61 \\
\hline
\end{tabular}

The presence of previously adsorbed $\mathrm{H}$ atoms on the surface causes a change to the site stability in such a way that the adsorption energy on Ti noticeably increases from a value of $0.73 \mathrm{eV}$ to a value of $-1.95 \mathrm{eV}$ when all surrounding four C-top sites are occupied with $\mathrm{H}$. The $\mathrm{H}$ atoms adsorbed in such a resulting pyramidal-like configuration exhibit two different heights above the surface: for the four $\mathrm{H}$ atoms on the C-top positions the height is $1.17 \AA$, whereas for the central Ti-top position the height of the $\mathrm{H}$ atom is $1.85 \AA$, see Figure 7.

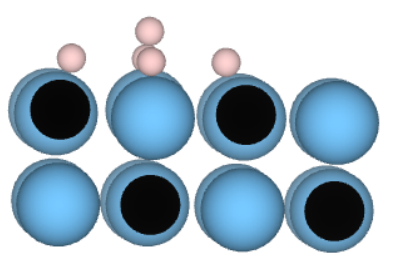

Side View

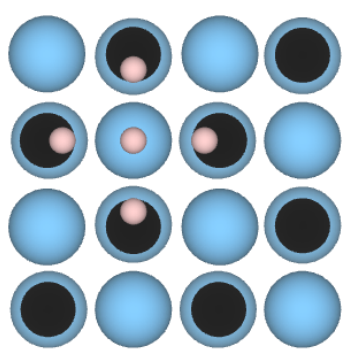

Top View

Figure 7. Pyramidal adsorption configuration formed with five adsorbed $H$ atoms. Left: Side view. Right: Top view. Blue balls represent Ti atoms, black balls are $\mathrm{C}$ atoms, pink balls are $\mathrm{H}$ atoms.

We also consider this mode of $\mathrm{H}$ adsorption for higher coverages. Specifically, the adsorption of a ninth $\mathrm{H}$ atom is evaluated when adsorbing on a full coverage of all four Ti-top and all four C-top sites of the supercell model of the $\mathrm{TiC}(001)$ surface. Here we compare the pyramidal Ti-Top mode with two other different positions for the subsequent $\mathrm{H}$ atom: on C-top 
forming a Kubas mode and subsurface, see Figure 8.

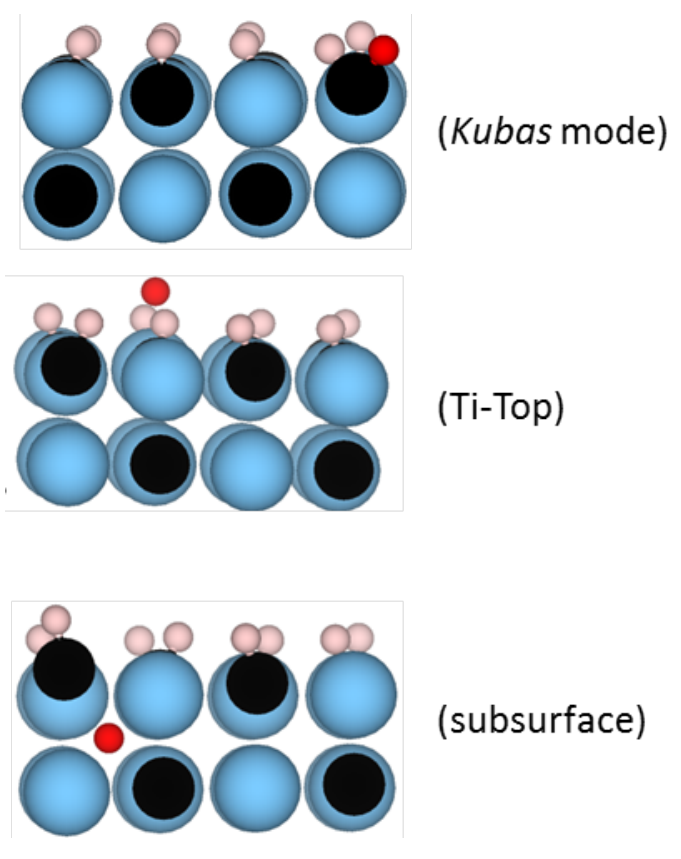

Figure 8. Side view of the three final positions evaluated where a ninth $\mathrm{H}$ atom is added to a slab that is pre-covered with eight adsorbed $\mathrm{H}$ atoms (colour code as in Figure 7, the added $\mathrm{H}$ atom is indicated in red).

The results for the three hydrogen adsorption modes evaluated show that the adsorption of the ninth $\mathrm{H}$ atom is again most favorable on a pyramidal Ti-top site, see Table 2. Notice that such a situation favors the occupancy of Ti atoms by $\mathrm{H}$ atoms with respect to $\mathrm{H}_{2}$ molecule in gas phase, with an adsorption energy of $-0.28 \mathrm{eV}$ as obtained at PBE-D3 level.

Table 2. Adsorption energies for the ninth $\mathrm{H}$ atom on $\mathrm{TiC}(001)$ on different sites as shown in Figure 8. All values are given in $\mathrm{eV}$.

\begin{tabular}{|c|c|c|c|}
\hline$E_{\text {ads }}^{H}$ & Kubas mode & Ti-Top & subsurface \\
\hline PBE & -1.94 & -2.43 & -1.12 \\
\hline
\end{tabular}


Another possibility to fill the surface is by direct molecular $\mathrm{H}_{2}$ physisorption, once all surface $\mathrm{C}$ atoms are covered by $\mathrm{H}$ adatoms, thus going the $\mathrm{H}$ coverage of over $1 \mathrm{ML}$ as found when exposing the sample to 1 Torr of $\mathrm{H}_{2}$. To analyze this situation, four physisorbed $\mathrm{H}_{2}$ molecules have been optimized on separated surface Ti atoms, ${ }^{13}$ see Figure 9 . These species have a mean adsorption energy of $-0.24 \mathrm{eV}$ (PBE-D3) with respect to the $\mathrm{H}_{2}$ reference, and are located $2.89 \AA$ above the surface plane, with an elongated $\mathrm{H}_{2}$ bond length $d(\mathrm{H}-\mathrm{H})$ of $0.76 \AA$.

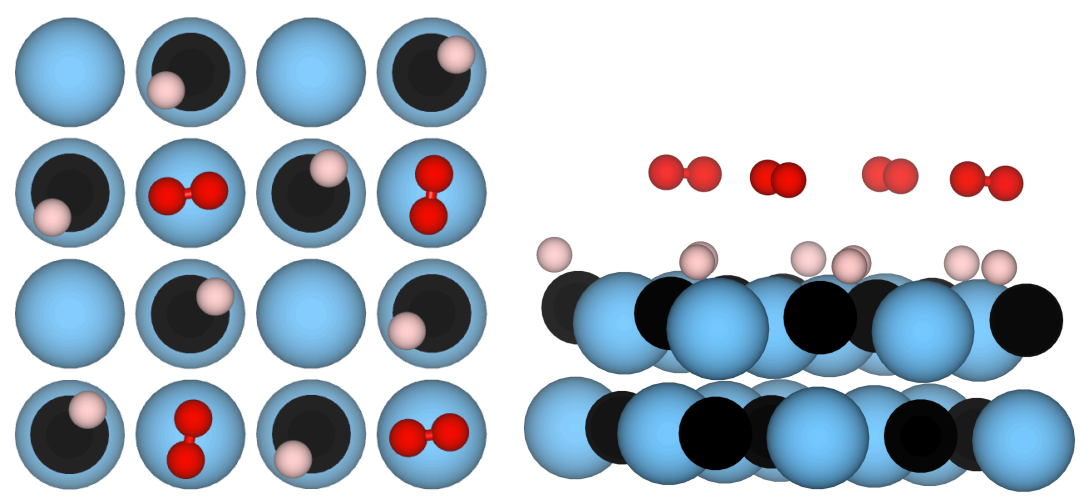

Figure 9. $\mathrm{H}_{2}$ molecules physisorbed on surface Ti-Top situations from top (left) and side (right) views. Blue balls represent $\mathrm{Ti}$ atoms, black balls are $\mathrm{C}$ atoms, and pink/red balls refer to $\mathrm{H}$ adatoms on surface C-Top or Ti-Top $\mathrm{H}_{2}$ physisorbed positions, respectively.

Altogether, the results allow us to provide an explanation of the experimental results. For the physisorbed $\mathrm{H}_{2}$ molecules, using Redhead equation, we estimate a TPD desorption range of 92-146 $\mathrm{K}$ which is much lower than the temperature range examined in the experiments of Figure 1, 320-420 K. Thus, the very high coverages of hydrogen seen in the experiments at temperatures above $300 \mathrm{~K}$ cannot be generated on a perfect surface of $\mathrm{TiC}(001)$ and must be associated with a reconstruction of the carbide. The exact nature of this reconstruction is unknown and, according to the XPS results in Figure 2, it involves the formation of $\mathrm{CH}_{\mathrm{x}}$ groups. In Figure 5, one can see desorption peaks for $\mathrm{H}_{2}$ at 325, 340, 365 and $405 \mathrm{~K}$. The feature at 405 
$\mathrm{K}$ is small and is probably associated with binding of hydrogen to defect sites of $\operatorname{TiC}(001)$. As mentioned above, the peak at $325 \mathrm{~K}$ is linked to a reconstruction of the carbide surface. Thus, only the peaks at 340 and $365 \mathrm{~K}$ may be associated with binding of hydrogen to an ideal $\mathrm{TiC}(001)$ surface. The calculations show that at small and medium coverages of $\mathrm{H}$ there is a sequential population of $\mathrm{C}$ and $\mathrm{Ti}$ atoms. In qualitative terms, the peak at $365 \mathrm{~K}$ probably correspond to binding of $\mathrm{H}$ to $\mathrm{C}$ sites, while the peak at $340 \mathrm{~K}$ could involve binding of $\mathrm{H}$ to $\mathrm{Ti}$ sites. Our predicted desorption temperatures $(212-325 \mathrm{~K}$ on $\mathrm{Ti}, 334-510 \mathrm{~K}$ on $\mathrm{C})$, estimated using the calculated adsorption energies and Redhead equation for limit cases of pre-exponential factor of $10^{13}-10^{8}$, respectively, can be somewhat smaller than the desorption temperatures in Figure 1, especially with the $10^{13}$ limit of the pre-exponential factor. This is expected due to the approximations in the DFT calculations and the Redhead equation. We note that, although our models provide insight into the relative static energetics of hydrogen adsorption modes, in such loosely bound and dynamic system different adsorption situations can co-exist and convert into each other, even in the course of the desorption process. Finally, the peak appearing at about 400 $\mathrm{K}$ with low intensity can be associated with $\mathrm{H}$ atom adsorption on defects on the surface such as steps or vacancies. In these positions, not considered here, the adsorption is assumed to be stronger, and the peak height small because of the low concentration of defects.

The TPD assignments are further supported by the experimental C $1 s$ XPS data shown in Figure 2, where a peak at $283 \mathrm{eV}$ is visible when the clean surface is annealed at a temperature of $500 \mathrm{~K}$, which indicates that the peak corresponds to Ti-C bonding. When the surface is exposed to $\mathrm{H}_{2}$ under UHV conditions at $300 \mathrm{~K}$, the TPD peaks at 365 and $340 \mathrm{~K}$ were observed producing an extra feature in the C $1 s$ XPS with a small shift of $0.1-0.2 \mathrm{eV}$ in the main peak. This result has been analyzed by estimating core-level shifts values obtained using the initial 
state model as well as the Janak-Slater model, as described in previous work. ${ }^{40,41}$ The present estimates reveal that a C-H configuration has a $\mathrm{C} 1 s$ binding energy only $0.27 \mathrm{eV}$ higher than the bare surface $\mathrm{C}$ in the carbide. In agreement with the trend seen in the XPS measurements for low coverages of $\mathrm{H}$ on $\mathrm{TiC}(001)$. Thus, the extra peak near $283.5 \mathrm{eV}$ in Figure 2 can be assigned to $\mathrm{CH}_{\mathrm{x}}$ groups on the basis of its binding energy, ${ }^{36}$ and proposed as $\mathrm{H}_{2}$ molecules trapped in interstitial sites of reconstructed $\mathrm{TiC}$ powders. ${ }^{34,35}$ On a flat $\mathrm{TiC}(001)$ surface, we found a very low adsorption energy for $\mathrm{H}_{2}$ but the $\mathrm{C}_{-} \mathrm{H}_{2}$ species displayed a $\mathrm{C} 1 s$ energy difference $1.23 \mathrm{eV}$ higher than bare surface $\mathrm{C}$ atoms. Thus, in the future when dealing with a reconstructed $\mathrm{TiC}(001)$, one must consider the possibility of $\mathrm{H}_{2}$ bound inside or on the modified carbide lattice.

\section{Conclusions}

Here, we demonstrate, both experimentally and theoretically, that $\mathrm{H}_{2}$ dissociation is possible on the $\mathrm{TiC}(001)$ surface. In addition, we reveal the nature of adsorbed $\mathrm{H}$ species once $\mathrm{H}_{2}$ has been dissociated. First, the $\mathrm{H}_{2}$ molecules adsorb on surface carbon atoms forming Kubas modes. Then, the $\mathrm{H}_{2}$ molecule dissociates although a small energy barrier of $0.49 \mathrm{eV}$. The dissociated $\mathrm{H}$ atoms are adsorbed first on top of the $\mathrm{C}$ atoms on the surface. Once adsorbed $\mathrm{H}$ atoms occupy $\mathrm{C}$ atoms on the surface, further $\mathrm{H}$ atoms can be stabilized adsorbed on the surface Ti atoms. Moreover, $\mathrm{H}_{2}$ molecules can be weakly adsorbed on surface titanium atoms, giving rise to a third type of adsorbed hydrogen. The sequence of occupation explains the TPD spectra at low coverage and is consistent with XPS spectra which show the existence of only one type of $H_{x}$ species on surface $\mathrm{C}$ atoms. A reconstruction of the $\mathrm{TiC}(001)$ surface makes possible the adsorption of large coverages of hydrogen. The present work provides a detailed description of the evolution of adsorbed $\mathrm{H}$ on $\mathrm{TiC}$ which is likely to hold for other TMCs with the same M:C ratio. 


\section{Acknowledgements}

The research carried out at Brookhaven National Laboratory was supported by the U.S. Department of Energy, Office of Science and Office of Basic Energy Sciences under contract No. DE-SC0012704. P.R. is grateful for financial support by BID and EN-SCN. The work was supported by Spanish MINECO/FEDER Grant CTQ2015-64618-R and in part by Generalitat de Catalunya Grants 2017SGR13 and XRQTC and by the NOMAD Center of Excellence Project receiving funding from the European Union Horizon 2020 Research and Innovation Programme, Grant Agreement 676580. F.V. thanks the Spanish MINECO for a Ramoń y Cajal research contract (RYC-2012-10129), F.I. acknowledges additional support through the ICREA Academia Award for Excellence in Research. J.J.P. thanks the Spanish MINECO for ayuda para la formación de doctores contract (BES-2016-78816). Financial support from Spanish MICIUN through the Excellence María de Maeztu program (grant MDM-2017-0767) is fully acknowledged.

Supporting Information Available: The explored positions for $\mathrm{H}$ adsorption on the $\mathrm{TiC}(001)$ surface (Figure $\mathrm{S} 1$ ), the $\mathrm{H}$ adsorption energies (Table $\mathrm{S} 1$ ), the explored positions for $\mathrm{H}_{2}$ adsorption on the $\mathrm{TiC}(001)$ surface (Figure $\mathrm{S} 2$ ), the $\mathrm{H}_{2}$ adsorption energies (Table $\mathrm{S} 2$ ), the order of hydrogen atoms addition on the $\mathrm{TiC}(001)$ surface (Figure $\mathrm{S} 3$ ), the adsorption energies for different $\mathrm{H}$ atoms coverage (Tables S3 S4). This material is available free of charge via the Internet at http://pubs.acs.org. 


\section{References}

(1) Kojima, I.; Miyazaki, E.; Inoue, Y.; Yasumori, I. Catalytic Activities of TiC, WC, and TaC for Hydrogenation of Ethylene. J. Catal. 1979, 59, 472-474.

(2) Fan, G.; Xiaojing, L.; Xu, C.; Jiang, W.; Zhang, Y.; Gao, D.; Bi, J.; Wang, Y. Palladium Supported on Titanium Carbide: A Highly Efficient, Durable, and Recyclable Bifunctional Catalyst for the Transformation of 4-Chlorophenol and 4-Nitrophenol. Nanomaterials 2018, 8, 141-154.

(3) Vidal, A. B.; Feria, L.; Evans, J.; Takahashi, Y.; Liu, P.; Nakamura, K.; Illas, F.; Rodriguez, J. A. $\mathrm{CO}_{2}$ Activation and Methanol Synthesis on Novel $\mathrm{Au} / \mathrm{TiC}$ and $\mathrm{Cu} / \mathrm{TiC}$ Catalysts. J. Phys. Chem. Lett. 2012, 3, 2275-2280.

(4) Rodriguez, J. A.; Evans, J.; Feria, L.; Vidal, A. B; Liu, P.; Nakamura, K.; Illas, F. CO 2 Hydrogenation on $\mathrm{Au} / \mathrm{TiC}, \mathrm{Cu} / \mathrm{TiC}$, and $\mathrm{Ni} / \mathrm{TiC}$ Catalysts: Production of $\mathrm{CO}$, Methanol, and Methane. J. Catal. 2013, 307, 162-169.

(5) Zhao, Y.; Dillon, A. C.; Kim, Y.; Heben, J.; Zhang, S. B. Self-Catalyzed Hydrogenation and Dihydrogen Adsorption on Titanium Carbide Nanoparticles. Chem. Phys. Lett. 2006, 425, 273277.

(6) Nguyen, J.; Glandut, N.; Jaoul, C.; Lefort, P. Electrochemical Hydrogen Insertion in Substoichiometric Titanium Carbide $\mathrm{TiC}_{0.6}$ : Influence of Carbon Vacancy Ordering. Langmuir 2013, 29, 12036-12042.

(7) Nguyen, J.; Glandut, N.; Jaoul, C.; Lefort, P. Hydrogen Insertion in Substoichiometric Titanium Carbide. Int. J. Hydrog. Energy 2015, 40, 8562-8570.

(8) Khidirov, I. Neutron Diffraction Study of Titanium Carbide $\mathrm{TiC}_{\mathrm{x}}$ Prepared from HydrogenContaining Titanium Powder. Russian J. Inorg. Chem. 2015, 60, 1263-1266.

(9) Osti, N. C.; Naguib, M.; Tyagi, M.; Gogotsi, Y.; Kolesnikov, A. I.; Mamontov, E. Evidence of Molecular Hydrogen Trapped in Two-Dimensional Layered Titanium Carbide-Based MXene. Phys. Rev. Mater. 2017, 1, 024004-1,6.

(10) Jimenez-Orozco, C.; Florez, E.; Moreno, A.; Liu, P.; Rodriguez, J. A. Systematic Theoretical Study of Ethylene Adsorption on $\delta$-MoC(001), TiC(001), and $\mathrm{ZrC}(001)$ Surfaces. $J$. Phys. Chem. C 2016, 120, 13531-13540. 
(11) Estreicher, S. K.; McAfee, J. L.; Fedders, P. A.; Pruneda, J. M.; Ordejón, P. The Strange Behavior of Interstitial $\mathrm{H}_{2}$ Molecules Si and GaAs. Physica B: Condens. Matter 2001, 308, $202-$ 205.

(12) Costanzo, F.; Silvestrelli, P. L.; Ancilotto, F. Physisorption, Diffusion, and Chemisorption Pathways of $\mathrm{H}_{2}$ Molecule on Graphene and on $(2,2)$ Carbon Nanotube by First Principles Calculations. J. Chem. Theory Comput. 2012, 8, 1288-1294.

(13) Florez, E.; Gomez, T.; Liu, P.; Rodriguez, J. A.; Illas, F. Hydrogenation Reactions on $\mathrm{Au} / \mathrm{TiC}(001)$ : Effects of Au-C Interactions on the Dissociation of $\mathrm{H}_{2}$. ChemCatChem 2010, 2 , 1219-1222.

(14) Skipper, C. V. J.; Hamaed, A.; Antonelli, D. M.; Kaltsoyannis, N. The Kubas Interaction in M(II) $(\mathrm{M}=\mathrm{Ti}, \mathrm{V}, \mathrm{Cr})$ Hydrazine-Based Hydrogen Storage Materials: a DFT Study. Dalton Trans. 2012, 41, 8515-8523.

(15) Posada-Pérez, S.; Viñes, F.; Valero, R.; Rodriguez, J. A.; Illas, F. Adsorption and Dissociation of Molecular Hydrogen on Orthorhombic $\beta-\mathrm{Mo}_{2} \mathrm{C}$ and Cubic $\delta$-MoC (001) Surfaces. Surf. Sci. 2017, 656, 24-32.

(16) Ding, H.; Fan, X.; Li, C.; Liu, X.; Jiang, D.; Wang, C. First-Principles Study of Hydrogen Storage in non-Stochiometric $\mathrm{TiC}_{\mathrm{x}}$. J. Alloys Compd. 2013, 551, 67-71.

(17) Ding, H.; Fan, X.; Chu, K.; Du, B.; Wang, J. The Influence of Stacking Faults on Hydrogen Storage in $\mathrm{TiC}_{\mathrm{x}}$. Int. J. Hydrog. Energy 2014, 39, 9262-9266.

(18) Hagen, J. Industrial Catalysis: A Practical Approach, Wiley-VCH Verlag GmbH \& Co, Weinheim, Germany, 2015.

(19) Spielmann, J.; Buch, F.; Harder, S. Early Main-Group Metal Catalysts for the Hydrogenation of Alkenes with $\mathrm{H}_{2}$. Angew. Chem. 2008, 120, 9576-9580.

(20) Rodriguez, J. A.; Liu, P.; Takahashi, Y.; Nakamura, K.; Viñes, F.; Illas, F. Desulfurization of Thiophene on $\mathrm{Au} / \mathrm{TiC}(001)$ : Au-C Interactions and Charge Polarization. J. Am. Chem. Soc. 2009, 131, 8595-8602.

(21) Frantz, P.; Didziulis, S. V. Detailed Spectroscopic Studies of Oxygen on Metal Carbide Surfaces. Surf. Sci. 1998, 412/413, 384-396. 
(22) Rodriguez, J. A.; Liu, P.; Dvorak, J.; Jirsak, T.; Gomes, J.; Takahashi, Y.; Nakamura, K. The Interaction of Oxygen with TiC(001): Photoemission and First-Principles Studies. J. Chem. Phys. 2004, 121, 465-474.

(23) Christmann, K.; Ertl, G.; Pignet, T. Adsorption of Hydrogen on a Pt(111) Surface. Surf. Sci. 1976, 54, 365-392.

(24) Quesne, M. G.; Roldan, A.; Leeuw, N. H.; Catlow, C. R. A. Bulk and Surface Properties of Metal Carbides: Implications for Catalysis. Phys. Chem. Chem. Phys. 2018, 20, 6905-6916.

(25) Kunkel, C.; Viñes, F.; Illas, F. Transition Metal Carbides as Novel Materials for $\mathrm{CO}_{2}$ Capture, Storage, and Activation. Energy Environ. Sci. 2016, 9, 141-144.

(26) Kresse, G.; Furthmüller, J. Efficient Iterative Schemes for Ab Initio Total Energy Calculations Using a Plane-Wave Basis Set. Phys. Rev. B. 1996, 54, 11169-11186.

(27) Perdew, J. P.; Burke, K.; Ernzerhof, M. Generalized Gradient Approximation Made Simple. Phys. Rev. Lett. 1996, 77, 3865-3868.

(28) Grimme, S.; Antony, J.; Ehrlich, S.; Krieg, S. A Consistent and Accurate Ab Initio Parametrization of Density Functional Dispersion Correction (DFT-D) for the 94 Elements H-Pu. J. Chem. Phys. 2010, 132, 154104.

(29) Blöch, P. E. Projector Augmented-Wave Method. Phys. Rev. B: Condens. Matter Mater. Phys. 1994, 50, 17953.

(30) Kresse, G.; Joubert, D. From Ultrasoft Pseudopotentials to the Projector Augmented-Wave Method. Phys. Rev. B: Condens. Matter Mater. Phys. 1999, 59, 1758.

(31) Monkhorst, H. J.; Pack, J. D. Special Points for Brillouin-Zone Integrations. Phys. Rev. B: Solid State 1976, 13, 5188.

(32) Henkelman, G.; Uberuaga, B. P.; Jónsson, H. A Climbing Image Nudged Elastic Band Method for Finding Saddle Points and Minimum Energy Paths. J. Chem. Phys. 2000, 113, 9901. (33) Reuter, K.; Scheffler, M. First-Principles Kinetic Monte Carlo Simulations for Heterogeneous Catalysis: Application to the CO Oxidation at $\mathrm{RuO}_{2}(110)$. Phys. Rev. B 2006, 73, 045433.

(34) Reuter, K.; Frenkel, D.; Scheffler, M. The Steady State of Heterogeneous Catalysis, Studied by First-Principles Statistical Mechanics. Phys. Rev. Lett. 2004, 93, 116105.

(35) Redhead, P. A. Thermal Desorption of Gases. Vacuum, 1962, 12, 203-211. 
(36) Pérez Escobar, D.; Wallaert, E.; Duprez, L.; Atrens, A.; Verbeken, K. Thermal Desorption Spectroscopy Study of the Interaction of Hydrogen with TiC Precipitates. Met. Mater. Int. 2013, 19, 741-748.

(37) Wei, F. G.; Tsuzaki, K. Quantitative Analysis on Hydrogen Trapping of TiC Particles in Steel. Metall. Mater. Trans. A 2006, 37, 331-353.

(38) Rodriguez, J. A.; Dvorak, J.; Jirsak, T. Chemistry of Thiophene on $\operatorname{Mo}(110), M_{0} C_{x}$ and MoS $_{\mathrm{x}}$ Surfaces: Photoemission Studies. Surf. Sci. 2000, 457, L413-L420.

(39) Tsuzuki, S.; Lüthi, H. P. Interaction Energies of Van der Waals and Hydrogen Bonded Systems Calculated Using Density Functional Theory: Assessing the PW91 Model. J. Chem. Phys. 2001, 114, 3949-3957

(40) Kunkel, C.; Viñes, F.; Ramírez, P. J.; Rodriguez, J. A.; Illas, F. Combining Theory and Experiment for Multitechnique Characterization of Activated $\mathrm{CO}_{2}$ on Transition Metal Carbide (001) Surfaces. J. Phys. Chem. C DOI: 10.1021/acs.jpcc.7b12227.

(41) Viñes, F.; Sousa, C.; Illas, F. On the Prediction of Core Level Binding Energies in Molecules, Surfaces and Solids. Phys. Chem. Chem. Phys. 2018, 20, 8403-8410. 
TOC Graphic

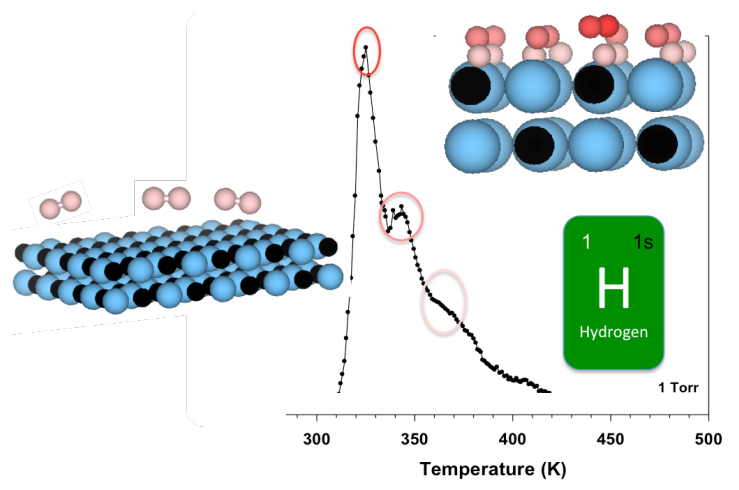

